\title{
Effects of Pretreatment and Drying on the Volatile Compounds of Sliced Solar-Dried Ginger (Zingiber officinale Roscoe) Rhizome
}

\author{
Roseline Esi Amoah (D), ${ }^{1}$ Faustina Dufie Wireko-Manu $\left(\mathbb{D},{ }^{2}\right.$ Ibok Oduro (D), \\ Firibu Kwesi Saalia $\left(\mathbb{D},{ }^{3}\right.$ William Otoo Ellis $\mathbb{D}^{2},{ }^{2}$ Alexander Dodoo $\mathbb{D}^{1},{ }^{1}$ Charles Dermont $\mathbb{D}^{\mathbb{D}},{ }^{4}$ \\ and Maame Ekua Manful $\mathbb{D D}^{5}$ \\ ${ }^{1}$ Ghana Standards Authority, Accra PMB MB 245, Ghana \\ ${ }^{2}$ Department of Food Science and Technology, Kwame Nkrumah University of Science and Technology, Kumasi, Ghana \\ ${ }^{3}$ Department of Food Process Engineering, P. O. Box LG 77, University of Ghana, Legon, Accra, Ghana \\ ${ }^{4}$ Graduate School of Agriculture and Bioengineering, JUNIA ISA, Lille, France \\ ${ }^{5}$ School of Food Science and Environmental Health, Technological University Dublin, Dublin, Ireland
}

Correspondence should be addressed to Roseline Esi Amoah; rosarchie@yahoo.com

Received 14 October 2021; Revised 2 December 2021; Accepted 10 December 2021; Published 8 January 2022

Academic Editor: Flora V. Romeo

Copyright ( $\odot 2022$ Roseline Esi Amoah et al. This is an open access article distributed under the Creative Commons Attribution License, which permits unrestricted use, distribution, and reproduction in any medium, provided the original work is properly cited.

\begin{abstract}
Ginger (Zingiber officinale Roscoe) rhizomes are mostly used as spice and medicine due to their high aroma intensity and medicinal bioactive compounds. However, the volatile compounds of ginger, partly responsible for its aroma and medicinal properties, can be affected by the pretreatment, drying method, and extraction processes employed. The objective of this study was to assess the effects of pretreatment and drying on the volatile compounds of yellow ginger variety at nine months of maturation. The effect of potassium metabisulfite (KMBS) and blanching pretreatment and drying on the volatile compounds of ginger using head space solid-phase microextraction with GCMS/MS identification (HS-SPME/GCMS/MS) was investigated. KMBS of concentrations 0.0 (control), $0.1,0.15,0.2$, and $1.0 \%$ and blanching at $50^{\circ} \mathrm{C}$ and $100^{\circ} \mathrm{C}$ were used for pretreatment and dried in a tent-like concrete solar (CSD) dryer and open-sun drying (OSD). The different concentrations of KMBS-treated fresh ginger rhizomes did not result in any particular pattern for volatile compound composition identification. However, the top five compounds were mostly sesquiterpenes. The 0.15\% KMBS-treated CSD emerged as the best pretreatment for retaining $\alpha$-zingiberene, $\beta$-cubebene, $\alpha$-farnesene, and geranial. The presence of $\beta$-cedrene, $\beta$-carene, and dihydro- $\alpha$-curcumene makes this study unique. The $0.15 \%$ KMBS pretreatment and CSD drying can be adopted as an affordable alternative to preserve ginger.
\end{abstract}

\section{Introduction}

Ginger (Zingiber officinale Roscoe) is a rhizome of the aromatic Zingiberaceae family mainly used as a spice [1]. It possesses strong pungent [2] and unique flavour components which are dominated by $\alpha$-zingiberene, $\alpha$-curcumene, $\alpha$-farnesene, $\beta$-sesquiphellandrene, $\beta$-bisabolene, geranial, and neral or Citral [3]. It is also used as herbal medicine due to its phenolic components such as gingerol, paradol, shogaol, and zingerone [4]. The amounts of these flavour components vary widely depending on the variety and processing methods used on the rhizome, especially the extraction and detection methods [5]. The subterranean environment of the rhizome before harvest subjects it to microbial contamination [6], and this requires pretreatment in addition to regular cleaning protocols to reduce the microbial load [1]. Several pretreatment methods involving the use of both chemical (sodium hydroxide $(\mathrm{NaOH})$ ), sodium chloride $(\mathrm{NaCL})$, potassium hydroxide $(\mathrm{KOH})$, sulfites, sodium metabisulfites (NaMBS), and potassium metabisulfites (KMBS)) and physical (thermal blanching (hot water, steam, superheated steam, etc.)) treatment have been applied to foods. In addition, nonthermal treatments such as ultrasound and freezing [1] have been applied to agricultural produce during processing such as drying with varying effects on the product characteristics and on consumer acceptability of the final product. Apart from reducing microbial load, 
pretreatment of agricultural produce is frequently done to minimize colour and or texture changes during drying [1]. Blanching of ginger has been done at a temperature of $70^{\circ} \mathrm{C}$ for a maximum duration of $30 \mathrm{~min}$ which reduced the pungent compound, gingerol, drastically [7]. The ginger volatile oils containing the flavour components are also reduced upon heat treatment just as the gingerol responsible for the pungency $[8,9]$. A pretreatment method that has frequently been used for agricultural produce including ginger without heat application is the use of dilute potassium metabisulfite (KMBS). Washing in dilute potassium metabisulfite has the additional advantage of reducing the microbial load as well as increasing the antioxidant activity of the product. It also partly preserves the ginger flavour compounds [10].

In addition to producing dried ginger of acceptable microbial safety, colour, and texture, the pretreatment and drying methods used must also preserve the volatile compounds. Flavour components in any food product are affected by the drying method $[9,11]$. Harsh drying methods that use elevated temperatures beyond $60^{\circ} \mathrm{C}$ may negatively affect the flavour of ginger $[8,9,12]$. Hence, the use of pretreatment and drying methods in the processing of ginger rhizomes must be carefully selected to achieve dried ginger which contains the dominant volatile compounds.

The methods for extraction and analyses of volatile compounds are also important. Solid-phase microextraction (SPME) is a simple, sensitive, and solvent-free technique which integrates sampling, isolation, and concentration of analytes from samples into a single-step process making it a fast and inexpensive sampling method [13]. Head space solid-phase microextraction (HS-SPME) is a method used in determining the flavour compounds of several products including ginger oil. Yang et al. [14] analysed oven-dried ginger by HS-SPME and detected chiefly $\alpha$-zingiberene (26.4\%), sesquiphellandrene $(10.2 \%), \beta$-phellandrene (10.0\%), camphene $(7.6 \%)$, geranial (6.6\%), $\alpha$-curcumene (6.0\%), and $\beta$-bisabolene (5.4\%). Similar work repeated with varying oven temperatures and drying methods, such as microwave drying, freeze drying, silica gel drying, and vacuum drying, detected similar top 5 volatile compounds but with varying concentrations $[3,11]$.

In most African countries and other tropical developing economies, industrial drying mostly involves the use of electrical energy which comes with additional cost, while most traditional approaches use open-sun drying. However, the solar drying approach could be an alternative process relative to the opensun drying. Solar drying has the advantage of reducing microbial load and environmental contamination associated with opensun drying [15]. Nonetheless, there is a paucity of data on the effects of solar drying and pretreatment on the flavour profile of ginger rhizomes. Thus, this study assessed the effects of pretreatment (soaking in dilute solutions of potassium metabisulfite (KMBS) and blanching) and drying on the volatile compounds of yellow ginger variety at nine months of maturation.

\section{Materials and Methods}

2.1. Source of Raw Materials. Eighty kilograms (80 kg) of fresh yellow ginger rhizomes at nine months of maturity were purchased from an outgrower in the ginger producing areas of Nkawie in the Ashanti region of Ghana. This is a town situated on latitude of $6^{\circ} 39^{\prime}$ $59.99^{\prime \prime} \mathrm{N}$ and longitude of $-1^{\circ} 48^{\prime} 59.99^{\prime \prime} \mathrm{W}$. The fresh ginger rhizomes were transported ( 6 hours) to the laboratory packed in a perforated nylon sack (to facilitate aeration).

2.2. Washing and Pretreating Fresh Ginger. The fresh raw ginger was soaked in water for two hours to remove the adhering sand/debris and washed vigorously three times each time with fresh water. The washed ginger rhizomes were sliced manually with a kitchen knife to a thickness of 3-5 mm. The sliced ginger was washed again in water. The sliced ginger was divided into seven parts; four parts were soaked separately in $0.1 \%, 0.15 \%, 0.2 \%$, and $1.0 \%$ potassium metabisulfite (KMBS) concentrations for 5 minutes. Another one part was soaked in water for 5 minutes serving as control. The final two parts were blanched at $50^{\circ} \mathrm{C}$ and $100^{\circ} \mathrm{C}$ temperatures for 5 minutes and 60 seconds, respectively. The pretreated sliced ginger rhizomes were drained separately and sampled for analyses of the volatile compounds' composition using HS-SPME/GCMS.

2.3. Drying of Fresh Washed Sliced Ginger. The remaining sliced ginger of each pretreatment was divided into two parts: one part was dried in the concrete solar dryer (CSD) (Figure 1) and the other part was dried in the open sun (OSD) for five days. The temperatures and humidity ranged $29.0-52.50^{\circ} \mathrm{C} ; 27-91.5 \% \mathrm{RH}$, and $28.0-55.5^{\circ} \mathrm{C} ; 29.5-90.5 \% \mathrm{RH}$ for the CSD and OSD, respectively, using a digital temperature-humidity data longer (HOBO pro v2 digital logger (Model U23-001, USA) (Appendix 1). The dried sliced ginger was further analysed for volatile compounds composition using HS-SPME/GCMS.

\subsection{Preparation of Ginger for the Head Space Solid-Phase Microextraction (HS-SPME) Analysis}

2.4.1. Pulverising and Milling of Ginger. All pretreated and untreated fresh ginger rhizomes were pulverised using a Moulinex blender (DPA141/35H-0517 R, France) for approximately $45 \mathrm{~s}$ and used for analysis. Pretreated and untreated sliced dried ginger were milled to pass through a sieve size of $1 \mathrm{~mm}$ using Moulinex mill (DPA141/35H$0517 \mathrm{R}$, France). The pulverized fresh and milled dried ginger samples were stored in plastic vials at $-18^{\circ} \mathrm{C}$ until used for analysis.

2.4.2. HS-SPME/Gas Chromatography-Mass Spectrometry (GC-MS) Analysis. The choice of an SPME fibre is essential because it may affect the type of compounds isolated. According to Ding et al. [11], a $100 \mu \mathrm{m}$ polydimethylsiloxane (PDMS) fibre proved to be the most useful in the extraction of ginger volatiles when compared to $30 \mu \mathrm{m}$ PDMS, $75 \mu \mathrm{m}$ carboxen polydimethylsiloxane (Carboxen PDMS), and 


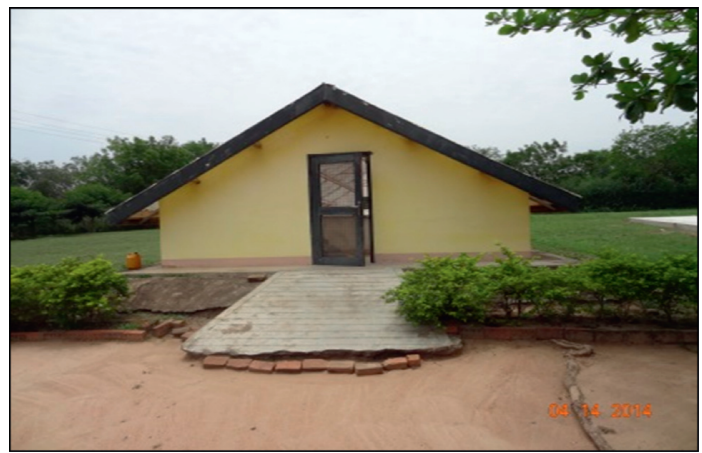

FIGURE 1: Pictorial representation of the concrete solar dryer (CSD).

TABLE 1: Major volatile compounds detected in pretreated sliced fresh yellow ginger rhizome.

\begin{tabular}{|c|c|c|c|c|c|c|c|c|}
\hline \multirow{3}{*}{ Retention time (min) } & \multirow{3}{*}{ Compound name } & \multicolumn{7}{|c|}{ Fraction (\%) } \\
\hline & & \multicolumn{5}{|c|}{ Potassium metabisulfite concentration } & \multicolumn{2}{|c|}{$\begin{array}{l}\text { Blanching } \\
\text { temperature }\end{array}$} \\
\hline & & Control & $0.10 \%$ & $0.15 \%$ & $1 \%$ & $0.20 \%$ & $50^{\circ} \mathrm{C}$ & $100^{\circ} \mathrm{C}$ \\
\hline 8.69 & $\alpha$-Pinene & & & & & 1.346 & & \\
\hline 8.98 & Camphene & 0.008 & 2.644 & 2.139 & 1.786 & 5.178 & 0.029 & \\
\hline 10.85 & $\alpha$-(E)-Sabinene hydrate & 0.015 & 4.136 & 3.417 & 3.706 & 6.196 & 0.050 & 0.099 \\
\hline 15.16 & Neral & & & & & 1.351 & & \\
\hline 15.18 & cis-Z- $\alpha$-bisabolene epoxide & & & 1.235 & & & & \\
\hline 15.19 & 2,6-Octadienal, 3,7-dimethyl (Z) & & & & & & 0.032 & \\
\hline 15.73 & Geranial & 0.008 & 2.647 & 2.231 & & 3.156 & 0.037 & 0.052 \\
\hline 15.74 & Dill-apiole & & & 2.298 & & & & \\
\hline 21.99 & $\alpha$-Curcumene & 0.014 & 4.192 & 32.920 & 4.294 & 16.827 & 0.488 & 0.665 \\
\hline 22.18 & Germacrene D & & 2.301 & & & & 0.030 & 0.051 \\
\hline 22.22 & $\alpha$-Cubebene & 0.015 & & & 2.600 & 3.519 & & \\
\hline 22.61 & $\alpha$-Zingiberene & & 32.983 & & 13.935 & & & \\
\hline 22.62 & Dihydro- $\alpha$-curcumene & 99.768 & & & 33.943 & 5.718 & & 97.898 \\
\hline 22.87 & $\alpha$-Farnesene & 0.040 & 12.149 & 11.103 & & & 0.154 & 0.266 \\
\hline 23.03 & $\alpha$-Bisabolene & 0.040 & 5.885 & & 8.089 & 10.686 & 98.821 & \\
\hline 23.07 & $\alpha$-Bisabolene & & & 7.648 & & & & \\
\hline 23.20 & (+)-Epibicyclosesquiphellandrene & & & & 0.983 & & & \\
\hline 23.48 & $\delta$-Carene & & & 15.441 & & & & \\
\hline 23.52 & $\alpha$-Cedrene & 0.046 & 15.614 & 4.171 & 16.213 & 21.543 & 0.201 & 0.159 \\
\hline 24.75 & $\gamma$-Gurjunene & 0.006 & 0.945 & & 0.723 & & & \\
\hline 26.17 & Zingerone & & & & & & & 0.037 \\
\hline
\end{tabular}

$65 \mu \mathrm{m}$ polydimethylsiloxane divinylbenzene (PDMS-DVD). The volatile components of ginger samples were extracted by head space solid-phase microextraction (HS-SPME) with a $100 \mu \mathrm{m}$ length PDMS fibre column (Supelco, USA). Preliminary analysis on this fibre gave an optimum temperature of $50^{\circ} \mathrm{C}$ at $23 \mathrm{~min}$ extraction time for $\alpha$-zingiberene, $\alpha$-farnesene, $\alpha$-curcumene, $\beta$-bisabolene, and $\beta$-cedrene.

Approximately $1 \mathrm{~g}$ of pulverised fresh ginger or milled dried ginger was weighed into a $10 \mathrm{~mL}$ amber glass vial. The fibre of $100 \mu \mathrm{m}$ diameter was manually inserted into the septum of the amber glass vial in the hot air oven at $50^{\circ} \mathrm{C}$ for $23 \mathrm{~min}$ and allowed for adsorption. The fibre was removed after adsorption and manually injected into the Gas Chromatography-Mass Spectrometry (GCMS) (Varian CP3800/3380, Walnut Creek, CA 94598 United States of America).
The Gas Chromatography coupled to Mass Spectrometry (GC-MS/MS) was used to identify the major compounds of the dried ginger. A Varian VF-1 ms capillary column $(30 \mathrm{~m} \times 0.25 \mathrm{~mm})$ attached to a Varian CP 3800 gas chromatography and a Varian Saturn 2000 spectrometry was used for the separation of the various components. The temperature gradient was as follows: $40^{\circ} \mathrm{C}(3 \mathrm{~min}), 130^{\circ} \mathrm{C}$ at $7^{\circ} \mathrm{C} / \mathrm{min}(3 \mathrm{~min}), 220^{\circ} \mathrm{C}$ at $3^{\circ} \mathrm{C} / \mathrm{min}(2 \mathrm{~min})$, and $260^{\circ} \mathrm{C}$ at $10^{\circ} \mathrm{C} / \mathrm{min}(5 \mathrm{~min})$. The flow rate of the helium gas was $1 \mathrm{~mL} /$ min and the fibre was introduced and injected at $230^{\circ} \mathrm{C}$ without split (split off). The compounds were identified by comparing the mass spectra of the chromatographs with built-in libraries with authentic standards. The relative percentage amount of each component was calculated by comparing its average peak area with the total area of the dry weight base. 
TABLE 2: Major volatile compounds detected in pretreated sliced solar dried yellow ginger rhizome.

\begin{tabular}{|c|c|c|c|c|c|c|c|c|}
\hline \multirow{3}{*}{ Retention time (min) } & \multirow{3}{*}{ Compound name } & \multicolumn{7}{|c|}{ Fraction (\%) } \\
\hline & & \multicolumn{5}{|c|}{ Potassium metabisulfite concentration } & \multicolumn{2}{|c|}{$\begin{array}{l}\text { Blanching } \\
\text { temperature }\end{array}$} \\
\hline & & $0.0 \%$ & $0.10 \%$ & $0.15 \%$ & $0.20 \%$ & $1 \%$ & $50^{\circ} \mathrm{C}$ & $100^{\circ} \mathrm{C}$ \\
\hline 8.69 & $\alpha$-Pinene & & & & & & 4.860 & 0.003 \\
\hline 8.98 & Camphene & 2.9685 & 1.32 & 0.005 & 0.007 & & & \\
\hline 10.82 & $\alpha$-Phellandrene & 4.3375 & & & & & & \\
\hline 10.85 & $\alpha$-(E)-Sabinene hydrate & & 2.96 & 0.011 & & & 3.034 & 0.006 \\
\hline 15.16 & Neral & & 1.70 & & & 0.002 & & 0.002 \\
\hline 15.18 & Z,Z,Z-4,6,9-Nonadecatriene & & & 0.005 & 0.007 & & & \\
\hline 15.19 & 2,6-Octadienal, 3,7-dimethyl (Z) & & & & & & 0.001 & \\
\hline 15.55 & Borneol & & & & & 0.001 & & \\
\hline 15.73 & Geranial & 2.5272 & 2.61 & 11.718 & 0.017 & 3.122 & 0.003 & 0.003 \\
\hline 15.74 & Dill-apiole & & & 0.005 & & & & \\
\hline 21.29 & $\alpha$-Farnesene & 0.0055 & & & & & & \\
\hline 21.99 & $\alpha$-Curcumene & 5.9425 & 4.89 & & 0.008 & 4.888 & 0.006 & 6.433 \\
\hline 22.18 & Germacrene D & 2.5849 & 2.86 & 0.081 & 0.115 & & 2.964 & \\
\hline 22.22 & $\alpha$-Cubebene & & & 31.813 & 44.703 & 3.462 & & 0.003 \\
\hline 22.61 & $\alpha$-Zingiberene & & & 19.377 & 0.028 & & & \\
\hline 22.62 & Dihydro- $\alpha$-curcumene & 39.719 & 41.40 & & & 41.017 & 43.035 & 44.523 \\
\hline 22.87 & $\alpha$-Farnesene & 15.536 & 16.20 & & & 17.073 & 16.459 & 17.137 \\
\hline 23.03 & $\alpha$-bisabolene & 9.2911 & 9.69 & & & 10.194 & 10.130 & 10.963 \\
\hline 23.07 & (6R,7 R)-bisabolene & & & 36.948 & 55.046 & & & \\
\hline 23.20 & Epibicyclosesquiphellandrene & & & & & 0.002 & & \\
\hline 23.52 & $\alpha$-Cedrene & 17.073 & 19.14 & & & 20.231 & 19.490 & 20.911 \\
\hline 24.75 & $\gamma$-Gurjunene & & & & 0.012 & & & \\
\hline 26.17 & Zingerone & & & & 0.004 & & & \\
\hline
\end{tabular}

\section{Results and Discussion}

The major volatile compounds identified are presented in Tables 1-3 and the HS-SPME/GCMS chromatograms in Appendix 2. The fresh control retained chiefly dihydro$\alpha$-curcumene (99.78\%), which is quite different compared to the compounds retained in the KMBS-pretreated fresh ginger rhizomes. Potassium metabisulfite-treated fresh ginger samples recorded $\alpha$-Curcumene, $\alpha$-Zingiberene, $\beta$-Cedrene, $\sigma$-Carene, $\alpha$-Bisabolene, and $\beta$-Bisabolene in different quantities as shown in Table 1.

The preservation of dihydro- $\alpha$-curcumene (99.78\%) as the major component in the fresh sample was totally different from the findings of similar work done [11] that detected compounds such as $\alpha$-zingiberene (28.12\%), $\beta$-citral (15.71\%), $\alpha$-farnesene $(6.90 \%), \beta$ - sesquiphellandrene $(7.65 \%)$, and $\alpha$-curcumene $(2.84 \%)$. The difference may be attributed to the differences in methodology and the source of the ginger. On the other hand, the samples treated with KMBS shared similar volatile compounds, especially $0.1 \%$ which had higher $\alpha$-Zingiberene (33\%) and $\alpha$-Farnesene $(12.15 \%)$ compared to the findings of Ding et al. [11]. Again, the retaining of high dihydro- $\alpha$-curcumene which has the same molecular weight and formula as $\alpha$-zingiberene in the control $(0.0 \% \mathrm{KMBS})$ may be due to harsh extraction conditions and possibly the temperature. $\alpha$-zingiberene is a sesquiterpene compound, unique to ginger, and was retained in $0.1 \%$ and $1.0 \%$ KMBS-treated fresh ginger in quantities of $33 \%$ and $13.94 \%$, respectively. The use of different concentrations of KMBS to pretreat the ginger rhizome did not show any particular pattern in the isolation of volatile compounds as the concentrations increased from $0.1 \%$ to $1.0 \%$; however, the presence of $\alpha$-zingiberene in higher quantities in the $0.1 \%$ KMBS-treated fresh ginger makes it more superior than the other concentrations and even the control of this study [16]. This shows that KMBS pretreatment had more diversified top five volatile compounds than the control.

The control of the fresh sample behaved similarly to the blanched samples by detecting one major volatile compound. The $100^{\circ} \mathrm{C}$ sample retained dihydro- $\alpha$-curcumene as the major component (97.9\%) just as the control, while the $50^{\circ} \mathrm{C}$ blanched sample had $\beta$-Bisabolene as the major component $(98.8 \%)$. This could affect the flavour of the blanched samples immensely because the compounds preserved in a ginger sample together contribute to its unique flavour. The blanching temperature of $50^{\circ} \mathrm{C}$ and $100^{\circ} \mathrm{C}$ were chosen based on the loss of gingerol beyond heat application of $60^{\circ} \mathrm{C}$ [12]; also the traditional processors boil the ginger rhizomes for $10 \mathrm{~min}$ to cure it before drying, hence $100^{\circ} \mathrm{C}$ to ascertain its effect. The preservation of one major dominant volatile compound in the fresh and blanched ginger rhizome of this study is similar to the findings of some work done [17] retaining zerumbone (85-87\%) as the single major volatile compound in fresh ginger rhizomes using the hydrodistillation method.

The effect of drying on the volatile compounds is shown in the differences between the fresh control and the control solar dried and open-sun-dried samples. The fresh control detected only dihydro- $\alpha$-curcumene $(99.8 \%)$ as shown in 
TABLE 3: Major volatile compounds detected in pretreated sliced open-sun dried yellow ginger rhizome.

\begin{tabular}{|c|c|c|c|c|c|c|c|c|}
\hline \multirow{3}{*}{ Retention time (min) } & \multirow{3}{*}{ Compound name } & \multicolumn{7}{|c|}{ Fraction $(\%)$} \\
\hline & & \multicolumn{5}{|c|}{ Potassium metabisulfite concentration } & \multicolumn{2}{|c|}{$\begin{array}{l}\text { Blanching } \\
\text { temperature }\end{array}$} \\
\hline & & Control & $0.10 \%$ & $0.15 \%$ & $0.20 \%$ & $1 \%$ & $50^{\circ} \mathrm{C}$ & $100^{\circ} \mathrm{C}$ \\
\hline 8.69 & $\alpha$-Pinene & & & & & & & \\
\hline 8.98 & Camphene & 2.1371 & 0.003 & 4.292 & 5.547 & 0.003 & 1.333 & 0.003 \\
\hline 10.89 & trans-4-Thujanol & 4.1767 & 5.338 & 5.893 & 8.300 & 0.006 & 1.992 & 4.866 \\
\hline 15.16 & Neral & 0.0014 & & & & & & \\
\hline 15.19 & 2,6-Octadienal, 3,7 -dimethyl (Z) & & 0.002 & & & & 0.001 & 0.001 \\
\hline 15.55 & Borneol & & & & & & & \\
\hline 15.73 & Geranial & 2.6124 & 0.003 & 0.003 & 0.004 & 0.003 & 0.001 & 0.002 \\
\hline 21.99 & $\alpha$-Curcumene & 5.9455 & 6.917 & 6.052 & 9.391 & 0.009 & 2.656 & 5.681 \\
\hline 22.18 & Germacrene D & & & & 0.004 & & & 0.003 \\
\hline 22.22 & $\alpha$-Cubebene & 2.7886 & 0.003 & 0.003 & & 0.004 & 1.149 & \\
\hline 22.61 & 1,3, 6,10-Dodeca tetraene,3, 7,11-trimethyl (Z, E)- & & & 40.171 & & & & \\
\hline 22.61 & $\alpha$-Zingiberene & 39.641 & & & & & & \\
\hline 22.62 & Dihydro- $\alpha$-curcumene & & 41.941 & & 6.326 & 58.085 & 17.546 & 42.915 \\
\hline 22.87 & $\alpha$-Farnesene & 15.57 & 16.653 & 15.126 & 25.222 & 0.023 & 62.812 & 16.487 \\
\hline 23.03 & $\alpha$-Bisabolene & 9.3904 & 10.091 & 9.633 & 15.520 & 14.397 & 4.250 & 10.215 \\
\hline 23.52 & $\alpha$-Cedrene & 17.72 & 19.032 & 18.813 & 29.651 & 27.440 & 8.252 & 19.811 \\
\hline 36.52 & Caryophyllene oxide & & & & 0.004 & 0.002 & & \\
\hline
\end{tabular}

Table 1, but the solar dried and open-sun-dried samples showed differences in the top 5 compounds detected. In the CSD samples, compounds detected were dihydro- $\alpha$-curcumene (39.72\%), $\alpha$-farnesene (15.54\%), $\beta$-bisabolene (9.69\%), $\alpha$-curcumene (5.94\%), and $\beta$-phellandrene (4.34\%) as shown in Table 2.

The detected compounds were different from the findings of one study that [14] reported mainly zingiberene, $\beta$-sesquiphellandrene, $\alpha$-farnesene, and $\beta$-bisabolene or $\alpha$-curcumene from air-dried ginger rhizome using the same fibre but different temperature and extraction time. The CSD samples also showed remarkable differences between the findings of some studies done $[3,11]$. They reported that drying increased or decreased certain volatile compounds affecting the overall flavour. Irrespective of the drying method employed in their study, similar dominant volatile compounds were isolated but in different quantities. Therefore, the observed differences in findings of this study relative to the other studies may be attributed to differences in extraction time and temperature [14] and/or variety of ginger. The results also showed differences between the CSD and OSD compounds, with about $40 \%$ of $\alpha$-zingiberene $(39.64 \%)$ being retained in OSD. The presence of this compound is the mark of quality making the difference quite remarkable. The following compounds were also isolated: $\alpha$-curcumene (5.95\%), $\alpha$-farnesene (15.57), $\beta$-bisabolene (9.39\%), and trans-4-thujanol (4.18\%) (Table 3). Thus, the characteristics of the OSD samples in terms of active compound composition were relatively more like the findings of Yang et al. [14], in retaining compounds such as $\alpha$-zingiberene $(53.12 \%)$, curcumene (4.9\%), $\alpha$-farnesene (8.61), $\beta$-bisabolene $(5.98 \%)$, and $\beta$-sesquiphellandrene (13.03\%), than when compared to the CSD samples. The differences were in the quantities which may be due to the variety and age of ginger rhizome or the differences in method of extraction as well as the drying methods used $[5,9,18]$. Apart from the top 5 compounds, CSD and OSD shared Camphene $(2.97 \% ; 2.145 \%)$ and Geranial $(2.53 \%$; $2.61 \%)$ in similar quantities.

The combination of pretreatment and drying affects the flavour compounds. KMBS is used in preserving the colour [19] of a product and to reduce and/or prevent microbial proliferation. However, the residual sulfur dioxide $\left(\mathrm{SO}_{2}\right)$ may affect the final flavour [20] underscoring the need for the application of the right concentration. In this study, the results showed that drying and pretreatment affected the volatile compounds isolated, which gives the unique final flavour of the dried ginger rhizome. The $0.1 \%$ and $1.0 \%$ KMBS-treated samples showed minor differences in the top 5 compounds isolated from the fresh and dried samples. These concentrations showed only one compound which was found in the fresh but not in the dried samples. $\alpha$-zingiberene (33\%) was isolated in the fresh 0.1\% KMBS samples but not in the dried samples. This may have decomposed to dihydro- $\alpha$-curcumene of $41.40 \%$ for CSD and of $41.94 \%$ for OSD, respectively (Bartley and Foley, 1994). Considering the detected volatile compounds for the $0.1 \%$ KMBS-treated samples, the dried samples had higher quantities than the fresh samples such as $\beta$-cedrene (15.6; $19.14 ; 19.03 \%), \alpha$-farnesene $(12.15 ; 16.20 ; 16.65 \%), \beta$-bisabolene $(5.89 ; 9.96 ; 10.09 \%)$, and $\alpha$-curcumene $(4.19 ; 4.89$; $6.92 \%$ ) for the fresh, CSD, and OSD, respectively. This agrees with some findings [11] that drying causes the reduction or increase of certain volatile compounds. For the 1.0\% KMBS samples, dihydro- $\alpha$-curcumene, $\beta$-cedrene, $\beta$ - bisabolene, and $\beta$-carene were common to the fresh and dried samples but in different quantities as shown in Tables 1-3. However, in this treatment, while $\alpha$-Zingiberene (33.9\%) was isolated in the fresh samples, $\alpha$-farnesene was peculiar to the dried samples. Both $\alpha$-zingiberene and $\alpha$-farnesene are 
sesquiterpenes with the same molecular formula and weight. Therefore, the effect of both KMBS and drying may account for this difference.

The volatile compounds of the $0.15 \%$ and $0.2 \%$ KMBS-treated samples showed a different pattern relative to the $0.1 \%$ and $1.0 \%$ KMBS-treated samples. The $0.15 \%$ and $0.2 \%$ KMBS-treated samples had more differences in the detected compounds. In these treatments, the fresh samples had more similar compounds to the OSD samples than the CSD. $\alpha$-Bisabolene was the only common compound present in the fresh $(7.65 \%)$ and CSD (36.95\%) for the 0.15\% KMBS-treated but in the $0.2 \%$ KMBS-treated samples, and the fresh samples shared no common compound with the CSD as shown in Tables 1 to 3 . The $0.15 \%$ fresh samples shared $\beta$-cedrene (4.17; $18.81 \%), \alpha$-farnesene $(11.10 ; 15.13 \%)$, and $\alpha$-curcumene $(32.9$; $6.05 \%)$ with the OSD, respectively. The $0.15 \%$ KMBS-treated CSD was the only KMBS dried sample that isolated $\alpha$-Zingiberene $(19.38 \%$ ) apart from the OSD dried control which is a mark of good quality ginger oil because it gives the specific ginger aroma [21] as well as compounds such as $\alpha$-bisabolene (36.95\%) and geranial (11.72\%) which give ginger rhizome the "citrus-like" flavor (Bartley and Foley, 1994). This study reports that $0.15 \%$ treated CSD sample is more superior to all dried samples irrespective of the drying method or pretreatment used. Even though the OSD control may be considered superior for having higher $\alpha$-zingiberene (39.64\%), research showed that this particular treatment had the highest yeast and mould contamination [1] which is unsafe for human consumption.

Blanching is a heat treatment that is used mostly to reduce drying time and microbial load. However, the high heat treatment regime for blanching can cause a change in the chemical composition of ginger rhizome, reducing both the pungency and flavour or altering it greatly [7]. Blanching and drying had a great effect on the volatile compounds. Blanching at $50^{\circ} \mathrm{C}$ for $5 \mathrm{~min}$ and $100^{\circ} \mathrm{C}$ for $60 \mathrm{~s}$ for the fresh samples detected only one major volatile compound which is $\alpha$-Bisabolene (98.82\%) and dihydro- $\alpha$-curcumene $(97.90 \%)$, respectively. On the other hand, the blanched, CSD, and OSD samples showed similar top 5 compounds, namely, dihydro- $\alpha$-curcumene, $\beta$-cedrene $\alpha$-farnesene, $\beta$-bisabolene, and $\alpha$-curcumene in spite of the temperature differences. The CSD $50^{\circ} \mathrm{C}$ samples showed similar compounds in almost equal quantities relative to the $\mathrm{CSD} 100^{\circ} \mathrm{C}$ samples (Table 2 ). This implies that the temperature of the blanched samples for CSD did not make much difference in the volatile compound composition as well as the quantities for both temperatures (dihydro- $\alpha$-curcumene $(43.04 ; 44.52 \%)$, $\beta$-cedrene $(19.49 ; 20.91 \%), \alpha$-farnesene $(16.45 ; 17.14 \%)$, $\beta$-bisabolene $(10.13 ; 10.96 \%)$, and $\alpha$-curcumene (4.89; $6.43 \%$ ) for $50^{\circ} \mathrm{C}$ and $100^{\circ} \mathrm{C}$, resp.). The pattern was different for the OSD samples for different temperatures as they gave different quantities of volatile compound (Table 3 ).

On the whole, none of the blanched samples, fresh or dried, retained $\alpha$-zingiberene, which is the mark of quality ginger oil using HS-SPME. The absence of $\alpha$-zingiberene from the blanched samples in this study reduces the quality of the samples and as such it is not a recommended choice of pretreatment for ginger.
The presence of $\beta$-cedrene, $\beta$-carene, and dihydro$\alpha$-curcumene isolated among the top 5 volatile compounds for the yellow variety makes this study unique from most work done using HS-SPME [3, 11, 14].

\section{Conclusion}

The effect of pretreatment with KMBS resulted in the appearance and disappearance of certain volatile compounds compared to the control. The different concentrations of KMBS treated with fresh ginger did not result in any particular pattern. However, $\alpha$-zingiberene was detected from $0.1 \%$ to $1.0 \%$ treated samples, which is the mark of quality ginger oil, making $0.1 \%$ superior to a higher $\alpha$-zingiberene fraction and recommended for fresh ginger preservation. Both blanched samples of $50^{\circ} \mathrm{C}$ and $100^{\circ} \mathrm{C}$ detected only dihydro- $\alpha$-curcumene and $\beta$-bisabolene, which may affect the overall flavour of the product. Drying alone showed OSD and CSD samples as having similar volatile compounds, yet OSD was seen to be better for detecting a higher $\alpha$-zingiberene fraction. On the other hand, the combination of pretreatment with KMBS and drying or blanching and drying showed the appearance and disappearance as well as increase and/or decrease of certain volatile compounds, which will determine the flavour of the dried ginger rhizomes. The $0.15 \%$ KMBS-treated CSD emerged as a better dried pretreatment for retaining $\alpha$-zingiberene, $\beta$-cubebene, $\alpha$-farnesene, and geranial. None of the blanched dried samples detected $\alpha$-zingiberene using HS-SPME/GCMS/MS and as such it is not a good pretreatment method for ginger rhizomes when flavour preservation is the objective. The tent-like concrete solar dryer can be adopted for commercial ginger drying with $0.15 \%$ KMBS pretreatment to preserve the volatile compounds of ginger. The limit of this study lies in the solar drying methods not being as controlled as mechanical dryers would have been. Nonetheless, the study was targeted at farmers and processors as a more affordable ginger preservation method than the mechanical dryers. It is expected that farmers and processors would adopt the use of this cost-effective method. It is also important to consider studies on suitable packaging materials in the future.

\section{Data Availability}

Data are deposited in a repository.

\section{Conflicts of Interest}

The authors declare no conflicts of interest for the content published in this manuscript.

\section{Acknowledgments}

The authors are very grateful to the management of ISA Lille Catholic University, Food Science Department, Lille, France, for making their laboratory facilities available for this research. This work was financially supported by the Ghana Standards Authority, Ghana. The head of the funding 
institution contributed to the editing and supervision of this study.

\section{Supplementary Materials}

Appendix 1: environmental conditions of the solar drying. Appendix 2: chromatograms of the volatile compounds of HS-SPME/GC-MS/MS analysis of ginger rhizome. (Supplementary Materials)

\section{References}

[1] R. E. Amoah, F. D. Wireko-Manu, I. Oduro, F. K. Saalia, and W. O. Ellis, "Effect of pretreatment on physicochemical, microbiological, and aflatoxin quality of solar sliced dried ginger (Zingiber officinale Roscoe) rhizome," Journal of Food Science and Nutrition, vol. 8, pp. 1-9, 2020.

[2] R. Offei-Okyne, J. Patterson, L. T. Walker, and M. Verghese, "Processing effects on phytochemical content and antioxidative potential of ginger," Zingiber officinale). Journal of Food and Nutrition Sciences, vol. 6, pp. 445-451, 2015.

[3] H. Baokang, W. Guowei, C. Zhiyong, and Q. Luping, "Effect of oven drying, microwave drying, and silica gel drying methods on the volatile components of ginger (Zingiber officinale Roscoe) by HS-SPME-GC-MS," Drying Technology: International Journal, vol. 30, no. 3, pp. 248-255, 2012.

[4] R. E. Amoah, S. Kalakandan, F. D. Wireko-Manu, I. Oduro, F. K. Saalia, and E. Owusu, "The effect of vinegar and drying (Solar and Open Sun) on the microbiological quality of ginger (Zingiber officinale Roscoe) rhizomes," Journal of Food Science and Nutrition, vol. 8, pp. 1-8, 2020.

[5] H. Yeh, C. Chuang, H. Chen, C. Wan, T. Chen, and L. Lin, "Bioactive components analysis of two various ginger (Zingiber officinale Roscoe) and antioxidant effect of ginger extracts," LWT- Journal of Food Science and Technology, vol. 55, pp. 329-334, 2014.

[6] E. E. Abdulkadir, A. A. Tahiya, N. Saif, Al-Bahry, and S. B. Charles, "Fungi and aflatoxins associated with spices in the Sultanate of Oman," Journal of Mycopathologia, vol. 155, pp. 155-160, 2002.

[7] H. Gan, E. Charters, R. Driscoll, and G. Srzednicki, "Effects of drying and blanching on the retention of bioactive compounds in ginger and turmeric," Journal of Horticulturae, vol. 3, p. 13, 2016.

[8] F. Irda, S. E. Waningish, and Sukrasno, "Heating effect of ginger (Zingiber officinale Roscoe) in content of volatile oil and oleoresin," International Journal of Research in Pharmacy and Science, vol. 5, no. 2, pp. 132-136, 2014.

[9] I. H. Sellami, W. A. Wannes, I. Bettaieb et al., "Qualitative and quantitative changes in the essential oil of Laurusnobilis L. leaves as affected by different drying methods," Journal of Food Chemistry, vol. 126, no. 2, pp. 691-697, 2011.

[10] A. Sangwan, A. Kawatra, and S. Sehgal, "Nutritional composition of ginger powder prepared using various drying methods," Journal of Food Science \& Technology, vol. 51, no. 9, pp. 2260-2262, 2012.

[11] S. H. Ding, K. J. An, C. P. Zhao, Y. H. Guo, and Z. F. Wang, "Effect of dying methods on volatiles of Chinese ginger, (Zingiber officinale Roscoe)," Journal of Food and Bioproduct Processing, vol. 90, pp. 515-524, 2012.

[12] D. A. Balladin, O. Headley, I. Chang-Yen, and D. R. Mcgaw, "High pressure liquid chromatographic analysis of the main pungent principles of solar dried West Indian ginger
(Zingiber officinale Roscoe)," Renewable Energy, vol. 13, no. 4, pp. 531-536, 1998.

[13] M. L. Bhavya and R. Ravi, "Development and validation of headspace Solid-Phase microextraction coupled with gas chromatography (HSSPME-GC) method for the analysis of zingiberzerumbet L," Natural Product Research, vol. 35, 2019.

[14] Z. Yang, W. Yang, Q. Peng et al., "Volatile phytochemical composition of the rhizome of ginger after extraction by headspace solid-phase microextraction, petroleum ether extraction and steam distillation extraction," Bangladesh Journal of Pharmacology, vol. 4, pp. 136-143, 2009.

[15] A. W. Deshmukh, M. N. Varma, C. K. Yoo, and K. L. Wasewar, "Investigation of solar drying of ginger (Zingiber officinale): empirical modelling, drying characteristics and quality studies," Chinese Journal of Engineering, vol. 2014, p. 7, Article ID 305823, 2014.

[16] A. Toure and Z. Xiaoming, "Gas chromatoghraphic analysis of volatile components of Guinean and Chinese ginger oils (Zingiber officinale) extracted by steam distillation," Journal of Agronomy, vol. 6, no. 2, pp. 350-355, 2007.

[17] H. R. Juliani, A. R. Koroch, J. E. Simon, J. Asante-Dartey, and D. Acquaye, "Chemistry and quality of fresh ginger varieties (Zingiber officinale) from Ghana," ISHS Acta Horticulturae, vol. 756, 2007.

[18] C. Gardelli, V. Papageorgiou, A. Mallouchos, K. Theodosis, and M. Komaitis, "Essential oil composition of Pistacia lentiscus L. and Myrtus communis L.: evaluation of antioxidant capacity of methalonic extracts," Journal of Food Chemistry, vol. 107, pp. 1120-1130, 2008.

[19] M. Okzan and B. Cemeroglu, "Desulphiting dried apricots by exposure to hot air flow," Journal of the Science of Food and Agriculture, vol. 82, pp. 1823-1828, 2002.

[20] O. I. Onyemaobi and G. O. Williams, "Cytogenetic effects of two food preservatives, sodium metabisulphite and sodium benzoate on the tips of Allium cepa Linn," Ife Journal of Science, vol. 14, no. 1, pp. 155-165, 2012.

[21] Z. Kamaliroostaa, L. Kamaliroosta, and A. H. Elhamirad, "Isolation and identification of ginger essential oil," Journal of Food Biosciences and Technology, vol. 3, pp. 73-80, 2013.

[22] G. S. Geeta and T. K. R. Reddy, "Aspergillus flavus Link and its occurrence in relation to other mycoflora on stored spices," Journal of Stored Products Research, vol. 26, no. 4, pp. 211-213, 1990.

[23] P. C. Onyenekwe and S. Hashimoto, "The composition of the essential oil of dried Nigerian ginger (Zingiber officinale Roscoe)," Journal of European Food Research and Technology, vol. 209, no. 6, pp. 407-410, 1999. 